\section{FENRETINIDE EXACERBATES ATHEROSCLEROSIS IN SPITE OF BENEFICIAL METABOLIC EFFECTS}

S. Manzini ${ }^{1}$, M. Busnelli ${ }^{1}$, C. Parolini ${ }^{1}$, E. Scanziani ${ }^{2}$, G. Chiesa $^{1}$ ${ }^{1}$ Department of Pharmacological and Biomolecular Sciences, Università degli Studi di Milano; ${ }^{2}$ Department of Veterinary Sciences and Public Health, Università degli Studi di Milano

Aim. Fenretinide, a synthetic retinoid derivative first investigated for cancer prevention and treatment, has been shown to ameliorate glucose tolerance and reduce body fat mass. These effects, together with its ability to inhibit ceramide synthesis, have suggested that fenretinide may display anti-atherosclerotic effects.

Methods. To this aim, 9-weeks-old apoE-KO (EKO) female mice were fed for 12 weeks a Western diet, without (control) or with $0.1 \%$ $\mathrm{w} / \mathrm{w}$ fenretinide ( $\mathrm{n}=20$ mice/group). Wild-type C57BL/6J (WT) mice were similarly enrolled to rule out possible genotype biase $(n=10)$. Mouse body weight, water and food intake were monitored throughout the study. Shortly before the end of the dietary treatment, plasma lipids and basal glucose levels were measured, and a glucose tolerance test was also performed in EKO. At sacrifice, heart and aorta were harvested to evaluate atherosclerosis development. In addition, liver, spleen, kidney, heart, white abdominal adipose and lung tissue underwent histological analysis.

Results. In EKO, fenretinide administration significantly lowered body weight and glucose levels, as well as plasma levels of tota cholesterol, triglycerides and phospholipids. In liver, fenretinide remarkably lessened the storage of glycogen and lipids driven by the Western diet. Unexpectedly, the treatment also led to abnormally enlarged spleens, with severe follicular atrophy and increased prevalence of extramedullary hematopoiesis. This latter finding was also observed in the liver, together with erythrophagocytosis. Heavy renal hemosiderin deposition was observed in fenretinide-treated mice. Finally, atherosclerosis development was markedly increased in fenretinide-treated EKO vs control at the aortic arch $(34.6 \pm 7.3 \%$ vs $26.1 \pm 5.8 \%,+33 \%)$, thoracic $(14.2 \pm 4.9 \%$ vs $4.9 \pm 2.1 \%,+190 \%)$ and abdominal aorta $(7.4 \pm 3.3 \%$ vs $3.3 \pm 1.8 \%$ $+124 \%)$. Fenretinide treatment to WT also caused body weight and abdominal fat reduction, with less severe spleen enlargement than in EKO. Biochemical and histological evaluations on WT are ongoing.

Conclusions. We first-time demonstrated that, despite beneficial metabolic effects, fenretinide treatment could be severely detrimental for atherosclerosis development.

\section{NORMALIZATION OF LIPOPROTEIN PROFILE DURING PREGNANCY IN LCAT DEFICIENCY}

A. Strazzella ${ }^{1}$, E. Hanna ${ }^{2}$, S. Simonelli ${ }^{1}$, A. Ossoli ${ }^{1}$, R. Mullan ${ }^{3}$, S. Chamney ${ }^{4}$, J Chestnutt ${ }^{2}$, F. Stewart ${ }^{5}$, L. Calabresi ${ }^{1}$ ${ }^{1}$ Centro E. Grossi Paoletti, Dipartimento di Scienze Farmacologiche e Biomolecolari, Università degli Studi di Milano;

${ }^{2}$ Department of Biochemistry;

${ }^{3}$ Renal Unit, Antrim Hospital, Northern Health and Social Care Trust, Antrim, UK,

${ }^{4}$ Department of Ophthalmology, Royal Victoria Hospital;

${ }^{5}$ Department of Genetics, Belfast City Hospital, Belfast Health and Social Care Trust, Belfast, UK

Aim. Subjects with genetic LCAT deficiency show marked alterations in plasma lipoprotein profile; among changes, the appearance of an abnormal lipoprotein, Lipoprotein X (LpX), seems to be involved in glomerulosclerosis development. Here we describe the normalization of lipoprotein profile during pregnancy in LCAT deficient woman.

Methods. A 29-year-old multigravida woman compound heterozygote with two LCAT gene mutations initially presented with bilateral corneal clouding, greatly reduced HDL-C and proteinuria (113.7 $\mathrm{mg} / \mathrm{mmol}$ creatinine). Fasting blood was collected at 22 weeks gestation and 14 weeks post-partum to assess possible changes during pregnancy.

Results. LCAT activity, cholesterol esterification rate, both undetectable, unesterified/total cholesterol ratio, and LCAT mass remained identical during pregnancy and post-partum. Total cholesterol, HDL-C, phospholipids, apoA-I and apoB increased during pregnancy. Her pregnancy was complicated by an hypertriglyceridaemia $(613 \mathrm{mg} / \mathrm{dL})$, which was more severe than is seen with normal physiological changes of pregnancy. Surprisingly, the level of proteinuria significantly improved during pregnancy $(13.9 \mathrm{mg} /$ mmol creatinine), despite stopping the ACE inhibitor, but it worsened post partum again $(131.6 \mathrm{mg} / \mathrm{mmol}$ creatinine). Lipoprotein analysis by FPLC and agarose gel electrophoresis of plasma revealed the presence LpX only in post-partum, whereas this abnormal particle disappeared during pregnancy.

Conclusion. $\mathrm{LpX}$ is an abnormal lipoprotein particle proposed as a major causative factor in the development of renal disease in carriers of LCAT deficiency. Its disappearance during pregnancy improves proteinuria in this woman and it is probably due to the severe increase of TG that are used, together with phospholipids, to produce VLDL. 